\title{
A cheap and simple passive sampler using silicone rubber for the analysis of surface water by gas chromatography-time of flight mass spectrometry
}

\author{
Yvette Naudé1,*, Peter Gorst-Allman² and Egmont Rohwer ${ }^{1}$ \\ 'University of Pretoria, Pretoria, South Africa \\ ${ }^{2}$ LECO Africa, Kempton Park, South Africa
}

\begin{abstract}
Water pollution events may arise rapidly, requiring a methodology that is easy to implement, fast to deploy, and sufficiently sensitive to detect the trace presence of hazardous contaminants. A cheap and easy to use silicone rubber (polydimethylsiloxane (PDMS)) miniature passive sampler is described. In order to test the methodology, pollutants were concentrated, in situ, from surface water in and around Pretoria, South Africa. The versatile sampler allowed for conventional and enhanced sensitivity, solvent-free analysis by comprehensive gas chromatography - time of flight mass spectrometry (GCxGC-TOFMS) and high resolution TOFMS (GC-HRT). Contaminants detected in surface water include caffeine, personal care products, pharmaceuticals, pesticides and polycyclic aromatic hydrocarbons.
\end{abstract}

Keywords: passive sampler, silicone rubber (PDMS) tubing, GCxGC-TOFMS, GC-HRT, surface water quality

\section{INTRODUCTION}

Water supply in South Africa is characterised by both achievements and challenges. In recent years, the country has made satisfactory progress with regard to improving access to water supply in urban areas. However, in some rural areas, women spend up to one-third of their time fetching water, and many rural dwellers are still without access to safe water sources and are compelled to take untreated water from rivers and dams. As a result of this situation, it is necessary to have a methodology which can be used for fast determination of pollutants, both organic and inorganic, which may possibly contaminate streams and wells. As water quality problems can arise very quickly, and can also be extremely short lived (e.g. spills or short- term releases into rivers), the methodology must be easy to implement, rapid to deploy, and must be sufficiently sensitive to detect the trace presence of harmful contaminants.

Spot water sampling gives chemical information specific to the moment of sampling and may fail to detect intermittent or transitory pollution. In contrast, passive sampling delivers a chemical profile representing days, weeks, or months depending on the duration of sampling. Furthermore, passive samplers accumulate analytes over time and thus provide enhanced sensitivity for trace level analytes compared to conventional bottle collection of water.

Silicone rubber (polydimethylsiloxane (PDMS)) functions as a hydrophobic solvent (Baltussen et al., 2002) and is therefore ideally suited for the in situ enrichment of persistent organic pollutants from water. Passive samplers are solvent extracted prior to instrumental analysis to obtain the trapped contaminants in an easily analysable form. Solvent extraction has the

This paper was originally presented at the 2014 Water Institute of Southern Africa (WISA) Biennial Conference, Mbombela, 25-29 May 2014.

* To whom all correspondence should be addressed.

욜 +27 12420 2517; e-mail: yvette.naude@up.ac.za disadvantage that it requires large amounts of often hazardous solvents and only microlitre amounts of the solvent extract are analysed. To address the disadvantages of bulk sample collection followed by solvent extraction, a cheap and easy to use passive sampler made from silicone rubber (PDMS) tubing, previously developed for solvent-free extraction of soil (Naudé et al., 2011; Naudé and Rohwer, 2012), was used. The passive sampler was applied to concentrate pollutants from surface water in and around Pretoria, South Africa. First results are reported.

\section{METHODOLOGY}

\section{Sampling sites}

Streams were sampled, in situ, at 3 different locations:

- Site 1: A small stream (Hartbeesspruit) flowing through the University of Pretoria (UP) recreational area, close to sports grounds, recreational braai (barbecue) areas and student residences (for 3 days)

- Site 2: The Moreletaspruit flowing through residential areas (for 3 days)

- Site 3: The Rietvleispruit running through the Rietvlei Nature Reserve (5 weeks).

The watercourses run through natural, industrial, and residential areas, and informal settlements. Sampling was performed from October 2013 to February 2014, in the summer, during the rainy season in Pretoria, South Africa.

\section{Miniature passive sampler}

A sampling loop was fashioned by taking a $10.5 \mathrm{~cm}(0.02 \mathrm{~g})$ length of silicone elastomer medical grade tubing $(0.64 \mathrm{~mm}$ OD $\times 0.3 \mathrm{~mm}$ ID, Sil-Tec Technical Products, Georgia, USA) and joining the ends by inserting a $1 \mathrm{~cm}$ piece of fused silica capillary column (250 $\mu \mathrm{m}$ ID) (Fig. 1). A loop arrangement prevents water from entering the PDMS tubing and aids ease of 


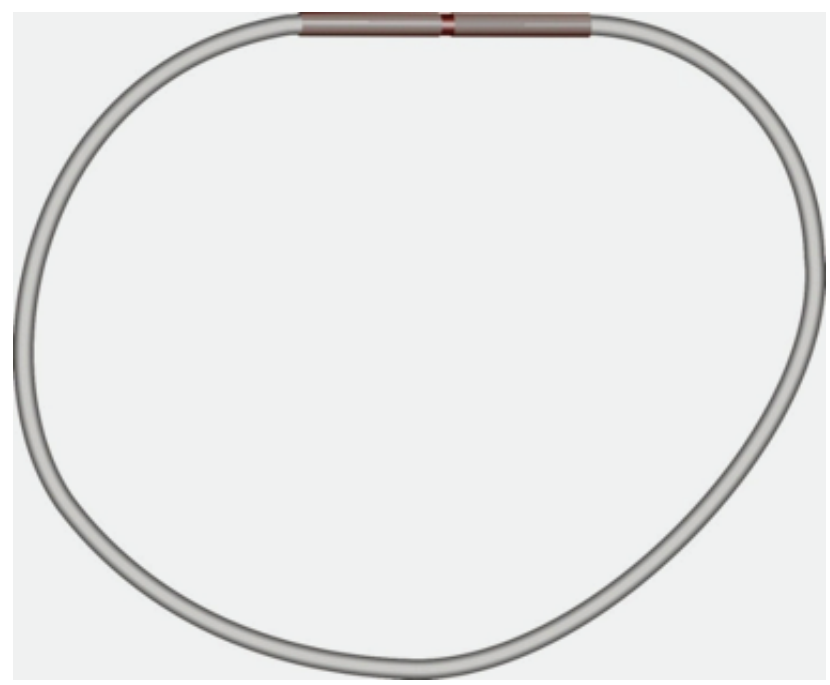

Figure 1

PDMS sampling loop: a $10.5 \mathrm{~cm}$ length of a silicone elastomer medical grade tubing joined at the ends by $1 \mathrm{~cm}$ piece of fused silica capillary column

handling. The sorption volume of the loop was $26 \mu \ell$ (Naudé, et al. 2011; Naudé and Rohwer, 2012). PDMS loops were inserted into a stainless steel mesh basket $(43 \mathrm{~mm} \times 30 \mathrm{~mm})($ Goldsmith \& Jewellery Supplies, Pretoria, South Africa). The sampling device was placed in situ, fully submerged in the watercourses described above.

After sampling, the PDMS loops were blotted with tissue paper and stored in an airtight glass container at $4^{\circ} \mathrm{C}$. The isolated analytes were desorbed from the PDMS loop either in the inlet liner of a GC inlet or in a Gerstel ${ }^{\mathrm{TM}}$ thermal desorber system. Alternatively, the analytes were solvent extracted from the PDMS loop with $1 \mathrm{~m} \ell n$-hexane (Merck, South Africa) followed by ultrasonication for $20 \mathrm{~min}$. One $\mu \ell$ of the solvent extract was injected $\left(250^{\circ} \mathrm{C}\right)$ in the splitless mode $(1 \mathrm{~min})$ for analysis.

\section{Thermal desorption with cooled injection}

After passive sampling, the PDMS loop was inserted into a 17.8 $\mathrm{cm}$ long glass desorption tube (4 $\mathrm{mm} \mathrm{ID}, 6.00 \mathrm{~mm} \mathrm{OD}$ ) from Gerstel $^{\text {TM }}$ (Chemetrix, Midrand, South Africa) for thermal desorption into a GCxGC-TOFMS. Compounds concentrated onto the PDMS sampler were thermally desorbed by heating the traps in a Gerstel ${ }^{\mathrm{TM}}$ thermal desorber system (TDS 3) from $30^{\circ} \mathrm{C}(3 \mathrm{~min})$ at $60^{\circ} \mathrm{C} / \mathrm{min}$ to $250^{\circ} \mathrm{C}(10 \mathrm{~min})$ with a desorption flow rate of $100 \mathrm{ml} / \mathrm{min}$ at a vent pressure of $117 \mathrm{kPa}$ (helium 5.0, Afrox, South Africa). The TDS transfer line temperature was $350^{\circ} \mathrm{C}$. The desorbed analytes were cryogenically focused at $-50^{\circ} \mathrm{C}$ using liquid nitrogen (Afrox, South Africa) and a cooled injection system (Gerstel CIS 4) with an empty baffled deactivated glass liner. After desorption, a splitless injection (purge on at $1 \mathrm{~min}$, purge flow $40 \mathrm{m \ell} / \mathrm{min}$, solvent vent mode) was performed by heating the CIS from $-50^{\circ} \mathrm{C}$ at $10^{\circ} \mathrm{C} / \mathrm{s}$ to $250^{\circ} \mathrm{C}$ and holding it there for the duration of the GC run.

\section{Thermal desorption in a GC inlet}

The PDMS loop was inserted into the glass inlet liner (splitless) of a GCxGC-TOFMS or GC-HRT and desorbed at $250^{\circ} \mathrm{C}$ with a splitless time of $1 \mathrm{~min}$.

\section{GCXGC-TOFMS}

Separation of compounds was performed on a LECO Pegasus $4 \mathrm{D}$ comprehensive gas chromatograph - time of flight mass spectrometer (GCxGC-TOFMS) including an Agilent 7890 GC (LECO Africa (Pty) Ltd., Kempton Park, South Africa). The system included a secondary oven and a dual stage modulator. Nitrogen gas (nitrogen generator) was used for both the cold jets and the hot jets. The gas for the cold jets was cooled by passing it through a dewar filled with liquid nitrogen. The column set consisted of a $30 \mathrm{~m} \times 0.25 \mathrm{~mm}$ ID $\times 0.25 \mu \mathrm{m}$ df Rxi 5SilMS (5\% phenyl, 95\% dimethylpolysiloxane) as the primary column $\left({ }^{1} \mathrm{D}\right)$, joined to a $1 \mathrm{~m} \times 0.25 \mathrm{~mm}$ ID $\times 0.25 \mu \mathrm{m}$ df Rxi17Sil MS secondary column ( $\left.{ }^{2} \mathrm{D}\right)$ (Restek, Bellefonte, PA, USA). The primary column was connected to the secondary column with a presstight column connector (Restek, Bellefonte, PA, USA). The primary oven temperature programme was $40^{\circ} \mathrm{C}(1$ $\min )$ at $10^{\circ} \mathrm{C} / \mathrm{min}$ to $320^{\circ} \mathrm{C}(2 \mathrm{~min})$. The $\mathrm{GC}$ run time was 31 $\mathrm{min}$. The secondary oven was offset by $+10^{\circ} \mathrm{C}$ from the primary oven. The modulator temperature was offset $15^{\circ} \mathrm{C}$ from the second oven temperature. The modulation period was $3 \mathrm{~s}$ with a hot pulse time of $0.6 \mathrm{~s}$. The carrier gas (helium 5.0, Afrox, South Africa) flow rate was $2 \mathrm{ml} / \mathrm{min}$ in the constant flow mode. The MS transfer line temperature was set at $300^{\circ} \mathrm{C}$. The ion source temperature was $230^{\circ} \mathrm{C}$, the electron energy was $70 \mathrm{eV}$ in the electron ionisation mode (EI+), the data acquisition rate was $100 \mathrm{spectra} / \mathrm{s}$, the mass acquisition range was $35-520 \mathrm{Da}$, and the detector voltage was set at $-1815 \mathrm{~V}$. Compounds were tentatively identified based on comparison of mass spectra (match quality $\geq 80 \%$ ) and experimental linear retention indices (RI) to those of the National Institute of Standards and Technology (NIST08) library and literature.

\section{GC-HRT}

The high-resolution TOFMS system was a Pegasus HRT (LECO Corporation, St Joseph, MI, USA) using Folded Flight Path technology (Verentchikov et al., 2005). The system had an Agilent 7890 GC (Agilent Technologies, Mississauga, ON) equipped with an Agilent 4513A autosampler. The column used was a $30 \mathrm{~m} \times 0.25 \mathrm{~mm}$ ID $\times 0.25 \mu \mathrm{m}$ df Rxi-5MS (5\% phenyl, 95\% dimethylpolysiloxane) (Restek, Bellefonte, PA, USA). The oven temperature programme was $35^{\circ} \mathrm{C}(1 \mathrm{~min})$ at $10^{\circ} \mathrm{C} / \mathrm{min}$ to $320^{\circ} \mathrm{C}$ ( $\left.2 \mathrm{~min}\right)$. The GC run time was $31.5 \mathrm{~min}$. The carrier gas (helium 5.0, Afrox, South Africa) flow rate was $2 \mathrm{ml} / \mathrm{min}$ in the constant flow mode. The MS transfer line temperature was set at $300^{\circ} \mathrm{C}$. The ion source temperature was $250^{\circ} \mathrm{C}$, the electron energy was $70 \mathrm{eV}$ in the electron ionisation mode (EI+), the data acquisition rate was 4 spectra/s, the mass acquisition range was $45-520 \mathrm{Da}$, and the extraction frequency was $1.8 \mathrm{kHz}$. Compounds were tentatively identified based on comparison of mass spectra to those of the NIST08 library. Mass accuracy was used to determine elemental composition which provided confirmation of the library search results.

\section{RESULTS AND DISCUSSION}

Dissolved compounds in surface water from 3 different sites were concentrated using PDMS loops as a passive sampler, and the results are depicted in Tables 1 to 6 . Rather than present the full list of compounds, data representative of different compound classes only are described in this publication. A full list of all the compounds detected is available on request from 
the authors. Selected compounds detected by GCxGC-TOFMS are reported in Tables 1 to 3 , and those detected by GC-HRT are reported in Tables 4 to 6 . The compounds described have been chosen to show the versatility of the trapping process for common chemicals found in the environment. In addition, they indicate a difference in the composition of the river water at the different sites.

GCxGC-TOFMS is a particularly effective tool for this type of analysis, due to its superior resolving capability based on both the boiling point and the polarity of compounds. The GCHRT provides excellent mass accuracy (routinely $<1 \mathrm{ppm}$ ) and this feature can be used to determine elemental composition which provides good confirmation of the library search results.

Distinct profiles are observed for each of the 3 sampling points (Fig. 2). PAHs (polycyclic aromatic hydrocarbons) are commonly found in the South African environment, especially close to informal settlements and recreational areas where open fires for warmth and cooking are common. Two of the rivers also flow close to small industrial areas, and this is indicated by the presence of several industrial chemicals (Fig. 2). Pesticides and herbicides are also found where the rivers are close to agricultural areas (Fig. 2). Personal care products are evident in the water which flows through residential areas and close to student residences and informal settlements. Caffeine, an ingredient in many beverages, food and medicinal products, was detected in water from the Moreletaspruit (Site 2) (Table 5). Since caffeine is excreted in urine, its presence in the aquatic environment serves as an indicator of human pollution (Ferreira, 2005).

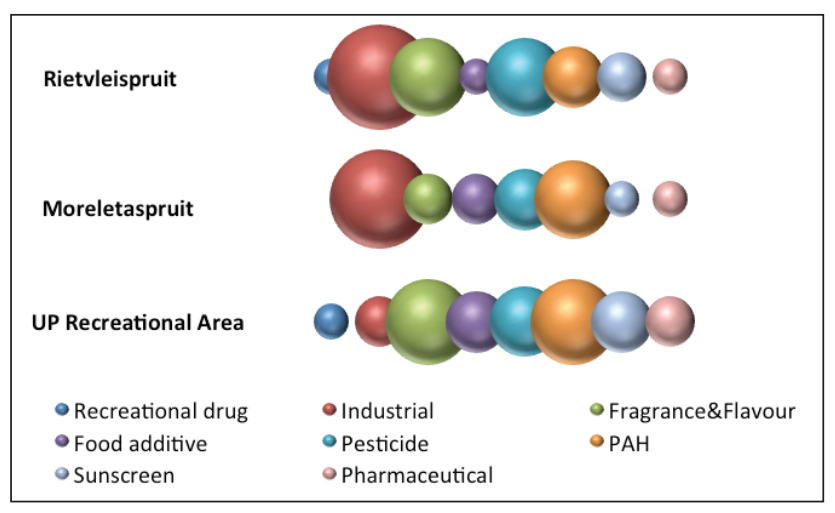

Figure 2

Compound classes detected in surface water from the 3 sites by GCXGCTOFMS and GC-HRT

Sample introduction into a GC-MSTOF was versatile in that the analytes were removed from the sampler device by either direct desorption in the GC inlet (GC-HRT), or by TDS (GCxGC-TOFMS), or by solvent extraction (GC-HRT). The advantage of thermal desorption, when compared to conventional liquid injection of microlitre amounts of a dilute extract, is enhanced sensitivity as the entire sample mass is transferred to the GC. A disadvantage is that thermal desorption is destructive. However, multiple loops are inserted into replicate stainless steel baskets and thus a sufficient number of loops are available for replicate runs.

\begin{tabular}{|c|c|c|c|c|c|c|c|}
\hline \multicolumn{8}{|c|}{$\begin{array}{c}\text { TABLE } 1 \\
\text { Selected compounds found in water from stream a }\end{array}$} \\
\hline Compound & Class & Similarity $^{1}$ & Formula & ${ }^{1} \mathrm{DRT}^{2}(\mathrm{~s})$ & ${ }^{2} \mathrm{D} \mathrm{RT}^{3}(\mathrm{~s})$ & ${ }^{1} \mathbf{D} \mathbf{R} \mathbf{e x p}_{\text {exp }}$ & ${ }^{1}$ D RI ${ }_{\text {Lit }}$ NIST08 \\
\hline Imidazole & Fungicide & 972 & $\mathrm{C}_{3} \mathrm{H}_{4} \mathrm{~N}_{2}$ & 448 & 0.69 & 1057 & $\begin{array}{l}1042, \\
1069\end{array}$ \\
\hline $\begin{array}{l}\text { Gamma-hydroxy- } \\
\text { butyrolactone }\end{array}$ & Recreational drug & 856 & $\mathrm{C}_{4} \mathrm{H}_{6} \mathrm{O}_{3}$ & 386 & 0.96 & 929 & 924 \\
\hline a-Phellandrene & Fragrance & 864 & $\mathrm{C}_{10} \mathrm{H}_{16}$ & 398 & 0.48 & 990 & $1004^{4}$ \\
\hline Maltol & Flavour & 935 & $\mathrm{C}_{8} \mathrm{H}_{10} \mathrm{O}$ & 498 & 0.76 & 1093 & 1091 \\
\hline Naphthalene & $\mathrm{PAH}$ & 917 & $\mathrm{C}_{10} \mathrm{H}_{8}$ & 564 & 0.81 & 1195 & 1197 \\
\hline Methenamine & Antibiotic & 838 & $\mathrm{C}_{6} \mathrm{H}_{12} \mathrm{~N}_{4}$ & 614 & 0.84 & 1269 & 1273 \\
\hline Caprolactam & Industrial & 899 & $\mathrm{C}_{6} \mathrm{H}_{11} \mathrm{NO}$ & 628 & 1 & 1290 & $\begin{array}{l}\text { Not available for } \\
\text { Rxi-5 phase }\end{array}$ \\
\hline Triacetin & Food and drug additive & 952 & $\mathrm{C}_{9} \mathrm{H}_{14} \mathrm{O}_{6}$ & 690 & 0.75 & 1379 & 1344 \\
\hline Piperonal & Fragrance and flavour & 892 & $\mathrm{C}_{8} \mathrm{H}_{6} \mathrm{O}_{3}$ & 692 & 0.93 & 1386 & $\begin{array}{l}\text { Not available for } \\
\text { Rxi-5 phase }\end{array}$ \\
\hline Ethylparaben & $\begin{array}{l}\text { Antifungal } \\
\text { Preservative } \\
\text { Food additive }\end{array}$ & 824 & $\mathrm{C}_{9} \mathrm{H}_{10} \mathrm{O}_{3}$ & 858 & 0.72 & 1602 & $\begin{array}{l}\text { Not available for } \\
\text { Rxi-5 phase }\end{array}$ \\
\hline Terbutylazine & Pesticide & 935 & $\mathrm{C}_{9} \mathrm{H}_{16} \mathrm{ClN}_{5}$ & 1008 & 0.85 & 1857 & $\begin{array}{l}\text { Not available for } \\
\text { Rxi-5 phase }\end{array}$ \\
\hline Phenanthrene & PAH & 954 & $\mathrm{C}_{14} \mathrm{H}_{10}$ & 1018 & 0.99 & 1815 & 1797 \\
\hline Anthrone & Tricyclic aromatic ketone & 888 & $\mathrm{C}_{14} \mathrm{H}_{10} \mathrm{O}$ & 1042 & 0.98 & 1908 & 1934 \\
\hline Homosalate & Sunscreen & 914 & $\mathrm{C}_{16} \mathrm{H}_{22} \mathrm{O}_{3}$ & 1066 & 0.7 & 1879 & $\begin{array}{l}\text { Not available for } \\
\text { Rxi-5 phase }\end{array}$ \\
\hline Thioxanthene & Pharmaceutical drug & 811 & $\mathrm{C}_{13} \mathrm{H}_{10} \mathrm{~S}$ & 1088 & 1.01 & 1977 & Not available \\
\hline Chlorpyrifos & Pesticide & 876 & $\mathrm{C}_{9} \mathrm{H}_{11} \mathrm{C}_{13} \mathrm{NO}_{3} \mathrm{PS}$ & 1128 & 0.87 & 1961 & 1950,1967 \\
\hline Oxybenzone & Sunscreen & 922 & $\mathrm{C}_{14} \mathrm{H}_{12} \mathrm{O}_{3}$ & 1164 & 1 & 2009 & $\begin{array}{l}\text { Not available for } \\
\text { Rxi-5 phase }\end{array}$ \\
\hline
\end{tabular}

${ }^{1}$ Spectral match quality (NIST08 library)

${ }^{2}$ First dimension retention time

${ }^{3}$ Second dimension retention time

${ }^{4}$ Babushok et al. (2011) 


\begin{tabular}{|c|c|c|c|c|c|c|c|}
\hline \multicolumn{8}{|c|}{$\begin{array}{l}\text { TABLE } 2 \\
\text { Selected compounds found in water from the Moreletaspruit by GCxGC-TOFMS }\end{array}$} \\
\hline Compound & Class & Similarity ${ }^{1}$ & Formula & ${ }^{1} \mathrm{DRT}^{2}(\mathrm{~s})$ & ${ }^{2} \mathrm{D} \mathrm{RT}^{3}(\mathrm{~s})$ & ${ }^{1} \mathbf{D} \mathbf{R} \mathbf{I}_{\text {exp }}$ & ${ }^{1} \mathrm{D} \mathrm{RI}_{\text {Lit }}$ NIST08 \\
\hline $\begin{array}{l}\text { 2,3-Butanedione } \\
\text { (Diacetyl) }\end{array}$ & Flavour & 801 & $\mathrm{C}_{4} \mathrm{H}_{6} \mathrm{O}_{2}$ & 118 & 0.35 & 618 & 608,621 \\
\hline 2-Furancarbonitrile & Sweetening agent & 927 & $\mathrm{C}_{5} \mathrm{H}_{3} \mathrm{NO}$ & 212 & 0.58 & 669 & Not available \\
\hline Ethylbenzene & $\begin{array}{l}\text { Industrial } \\
\text { Petrochemical }\end{array}$ & 877 & $\mathrm{C}_{8} \mathrm{H}_{10}$ & 264 & 0.49 & 812 & 833 \\
\hline p-Xylene & $\begin{array}{l}\text { Industrial } \\
\text { Petrochemical }\end{array}$ & 944 & $\mathrm{C}_{8} \mathrm{H}_{10}$ & 272 & 0.5 & 823 & 848 \\
\hline Styrene & Industrial & 935 & $\mathrm{C}_{8} \mathrm{H}_{8}$ & 290 & 0.55 & 847 & 857 \\
\hline Hexylene glycol & $\begin{array}{l}\text { Industrial } \\
\text { Washing/cleaning } \\
\text { products }\end{array}$ & 917 & $\mathrm{C}_{6} \mathrm{H}_{14} \mathrm{O}_{2}$ & 330 & 0.58 & 900 & 892 \\
\hline Benzaldehyde & $\begin{array}{l}\text { Industrial } \\
\text { Flavour }\end{array}$ & 937 & $\mathrm{C}_{7} \mathrm{H}_{6} \mathrm{O}$ & 356 & 0.73 & 935 & 926 \\
\hline Limonene & $\begin{array}{l}\text { Flavour } \\
\text { Personal care }\end{array}$ & 939 & $\mathrm{C}_{10} \mathrm{H}_{16}$ & 420 & 0.5 & 1020 & 1017 \\
\hline Biphenyl & $\begin{array}{l}\text { Industrial } \\
\text { Pesticide }\end{array}$ & 913 & $\mathrm{C}_{12} \mathrm{H}_{10}$ & 726 & 0.8 & 1427 & 1425 \\
\hline Fluorene & PAH & 931 & $\mathrm{C}_{13} \mathrm{H}_{10}$ & 882 & 0.89 & 1634 & 1604 \\
\hline 9H-Xanthene & Fungicide & 863 & $\mathrm{C}_{13} \mathrm{H}_{10} \mathrm{O}$ & 908 & 0.85 & 1669 & 1661 \\
\hline Dibenzothiophene & Petroleum & 896 & $\mathrm{C}_{12} \mathrm{H}_{8} \mathrm{~S}$ & 998 & 0.99 & 1788 & 1766 \\
\hline $\begin{array}{l}\text { 2-Ethylhexyl } \\
\text { salicylate }\end{array}$ & Sunscreen & 854 & $\mathrm{C}_{15} \mathrm{H}_{22} \mathrm{O}_{3}$ & 1026 & 0.65 & 1826 & Not available \\
\hline Fluoranthene & PAH & 938 & $\mathrm{C}_{16} \mathrm{H}_{10}$ & 1186 & 1.07 & 2038 & 2039 \\
\hline Pyrene & $\mathrm{PAH}$ & 932 & $\mathrm{C}_{16} \mathrm{H}_{10}$ & 1216 & 1.14 & 2078 & 2072 \\
\hline
\end{tabular}

${ }^{1}$ Spectral match quality (NIST08 library)

${ }^{2}$ First dimension retention time

${ }^{3}$ Second dimension retention time

TABLE 3

Selected compounds found in water from the Rietvleispruit by GCxGC-TOFMS

\begin{tabular}{|c|c|c|c|c|c|c|c|}
\hline Compound & Class & Similarity $^{1}$ & Formula & ${ }^{1} \mathbf{D ~ R T}^{2}$ (s) & ${ }^{2} \mathrm{D} \mathrm{RT}^{3}(\mathrm{~s})$ & ${ }^{1} \mathbf{D} \mathbf{R} \mathbf{e x p}_{\text {exp }}$ & ${ }^{1} \mathrm{D} \mathrm{RI}_{\text {Lit }}$ NIST08 \\
\hline 1,3-Butanediol & $\begin{array}{l}\text { Solvent } \\
\text { Food flavour agents }\end{array}$ & 912 & $\mathrm{C}_{4} \mathrm{H}_{10} \mathrm{O}_{2}$ & 286 & 0.61 & 842 & Not available \\
\hline Dimethyl trisulfide & $\begin{array}{l}\text { Bacterial } \\
\text { decomposition }\end{array}$ & 868 & $\mathrm{C}_{2} \mathrm{H}_{6} \mathrm{~S}_{3}$ & 362 & 0.68 & 943 & 950 \\
\hline Phenol & $\begin{array}{l}\text { Industrial } \\
\text { Herbicides } \\
\text { Pharmaceutical } \\
\text { Petroleum }\end{array}$ & 930 & $\mathrm{C}_{6} \mathrm{H}_{6} \mathrm{O}$ & 372 & 0.66 & 956 & 961 \\
\hline Benzonitrile & Industrial & 861 & $\mathrm{C}_{7} \mathrm{H}_{5} \mathrm{~N}$ & 376 & 0.76 & 961 & 951,986 \\
\hline Benzyl chloride & Industrial & 846 & $\mathrm{C}_{7} \mathrm{H}_{7} \mathrm{Cl}$ & 404 & 0.68 & 999 & 1002 \\
\hline Phenylethyl alcohol & Fragrance & 944 & $\mathrm{C}_{8} \mathrm{H}_{10} \mathrm{O}$ & 498 & 0.76 & 1123 & 1113 \\
\hline Quinoline & $\begin{array}{l}\text { Coal } \\
\text { Environmental } \\
\text { contaminant }\end{array}$ & 923 & $\mathrm{C}_{9} \mathrm{H}_{7} \mathrm{~N}$ & 612 & 0.88 & 1275 & 1247 \\
\hline Hydroquinone & $\begin{array}{l}\text { Skin whitening } \\
\text { (banned) }\end{array}$ & 809 & $\mathrm{C}_{6} \mathrm{H}_{6} \mathrm{O}_{2}$ & 632 & 0.91 & 1302 & 1327 \\
\hline (E)-Cinnamaldehyde & $\begin{array}{l}\text { Flavour and fragrance } \\
\text { Fungicide }\end{array}$ & 916 & $\mathrm{C}_{9} \mathrm{H}_{8} \mathrm{O}$ & 638 & 0.86 & 1310 & 1323 \\
\hline Phthalimide & Plastic & 949 & $\mathrm{C}_{8} \mathrm{H}_{5} \mathrm{NO}_{2}$ & 792 & 1.05 & 1514 & Not available \\
\hline $\begin{array}{l}\text { Diethyltoluamide } \\
\text { (DEET) }\end{array}$ & Insect repellent & 932 & $\mathrm{C}_{12} \mathrm{H}_{17} \mathrm{NO}$ & 870 & 0.84 & 1618 & $\begin{array}{l}\text { Not available for } \\
\text { Rxi-5 phase }\end{array}$ \\
\hline
\end{tabular}


TABLE 3 (continued)

Selected compounds found in water from the Rietvleispruit by GCxGC-TOFMS

\begin{tabular}{|c|c|c|c|c|c|c|c|}
\hline Compound & Class & Similarity $^{1}$ & Formula & ${ }^{1} \mathrm{D} \mathrm{RT}^{2}(\mathbf{s})$ & ${ }^{2} \mathrm{D} \mathrm{RT}^{3}(\mathrm{~s})$ & ${ }^{1} \mathbf{D} \mathbf{R} \mathbf{I}_{\exp }$ & ${ }^{1} D$ RI $_{\text {Lit }}$ NIST08 \\
\hline Fluorene & PAH & 934 & $\mathrm{C}_{13} \mathrm{H}_{10}$ & 882 & 0.88 & 1634 & 1595 \\
\hline Terbuthylazine & Herbicide & 902 & $\mathrm{C}_{9} \mathrm{H}_{16} \mathrm{ClN}_{5}$ & 1006 & 0.85 & 1799 & 1778 \\
\hline Metalochlor & Herbicide & 853 & $\mathrm{C}_{15} \mathrm{H}_{22} \mathrm{ClNO}_{2}$ & 1124 & 0.86 & 1956 & 1968 \\
\hline Oxybenzone & Sunscreen & 889 & $\mathrm{C}_{14} \mathrm{H}_{12} \mathrm{O}_{3}$ & 1162 & 1 & 2007 & $\begin{array}{l}\text { Not available for } \\
\text { Rxi-5 phase }\end{array}$ \\
\hline
\end{tabular}

${ }^{1}$ Spectral match quality (NIST08 library)

${ }^{2}$ First dimension retention time

${ }^{3}$ Second dimension retention time

TABLE 4

Selected compounds found in water from stream at UP recreational area (Hartbeesspruit) by GCHRT

\begin{tabular}{|c|c|c|c|c|c|}
\hline Compound & Class & $\mathbf{R T}^{1}(\mathbf{s})$ & Similarity ${ }^{2}$ & Formula & $\begin{array}{c}\text { Mass accuracy } \\
\text { (ppm) }\end{array}$ \\
\hline 2-Furanone & $\mathrm{O}$ & 332 & 548 & $\mathrm{C}_{4} \mathrm{H}_{4} \mathrm{O}_{2}$ & -0.93 \\
\hline 2-Pentylfuran & Flavour & 405.3 & 822 & $\mathrm{C}_{9} \mathrm{H}_{14} \mathrm{O}$ & -0.15 \\
\hline 1,3,5-Trimethylbenzene & Combustion $\mathrm{VOC}^{3}$ & 407.3 & 889 & $\mathrm{C}_{9} \mathrm{H}_{12}$ & -0.08 \\
\hline 1,2-Dichlorobenzene & $\mathrm{Cl}$ & 425.4 & 783 & $\mathrm{C}_{6} \mathrm{H}_{4} \mathrm{Cl}_{2}$ & -0.44 \\
\hline Limonene & $\begin{array}{l}\text { Personal care } \\
\text { Flavour }\end{array}$ & 442.3 & 638 & $\mathrm{C}_{10} \mathrm{H}_{16}$ & 0.01 \\
\hline 1-Ethyl-2,4-dimethylbenzene & Aromatic & 488.8 & 861 & $\mathrm{C}_{10} \mathrm{H}_{14}$ & -0.53 \\
\hline o-Cymene & Personal care & 495 & 623 & $\mathrm{C}_{10} \mathrm{H}_{14}$ & 0.08 \\
\hline Dodecane & Hydrocarbon & 597.3 & 712 & $\mathrm{C}_{12} \mathrm{H}_{26}$ & 1.89 \\
\hline Benzothiazole & S & 622.8 & 853 & $\mathrm{C}_{7} \mathrm{H}_{5} \mathrm{NS}$ & -0.44 \\
\hline Indole & $\mathrm{N}$ & 680.5 & 632 & $\mathrm{C}_{8} \mathrm{H}_{7} \mathrm{~N}$ & -0.7 \\
\hline Acenaphthene & PAH & 832.3 & 653 & $\mathrm{C}_{12} \mathrm{H}_{10}$ & -0.55 \\
\hline Dibenzofuran & $\mathrm{O}$ & 855.5 & 857 & $\mathrm{C}_{12} \mathrm{H}_{8} \mathrm{O}$ & -0.32 \\
\hline 1H-Phenalene & $\mathrm{PAH}$ & 904 & 571 & $\mathrm{C}_{13} \mathrm{H}_{10}$ & 0.74 \\
\hline Bifenthrin & Pesticide & 932.3 & 603 & $\mathrm{C}_{14} \mathrm{H}_{13}$ & -0.48 \\
\hline Benzophenone & Sunscreen additive & 934 & 820 & $\mathrm{C}_{13} \mathrm{H}_{10} \mathrm{O}$ & -1.08 \\
\hline Dibenzothiophene & S & 1018.5 & 840 & $\mathrm{C}_{12} \mathrm{H}_{8} \mathrm{~S}$ & 0.27 \\
\hline Anthracene & $\mathrm{PAH}$ & 1035.8 & 925 & $\mathrm{C}_{14} \mathrm{H}_{10}$ & 0.05 \\
\hline Pyrene & PAH & 1204.3 & 943 & $\mathrm{C}_{16} \mathrm{H}_{10}$ & 1.9 \\
\hline Benz[a]anthracene & $\mathrm{PAH}$ & 1411 & 912 & $\mathrm{C}_{18} \mathrm{H}_{12}$ & -0.05 \\
\hline Perylene & $\mathrm{PAH}$ & 1550.3 & 806 & $\mathrm{C}_{20} \mathrm{H}_{12}$ & 0.23 \\
\hline
\end{tabular}

${ }^{1}$ Retention time

${ }^{2}$ Spectral match quality (NIST08 library)

${ }^{3}$ Volatile organic compound

\begin{tabular}{|l|l|c|c|c|c|}
\hline \multicolumn{7}{|l|}{ TABLE 5 } \\
Selected compounds found in water from the Moreletaspruit by GC-HRT \\
\hline Compound & Class & RT (s) & Similarity & Formula & $\begin{array}{c}\text { Mass accuracy } \\
\text { (ppm) }\end{array}$ \\
\hline 2-Cyclohexen-1-one & $\mathrm{O}$ & 336.8 & 785 & $\mathrm{C}_{6} \mathrm{H}_{8} \mathrm{O}$ & 0.41 \\
\hline Phenol & Industrial chemical & 391.8 & 774 & $\mathrm{C}_{6} \mathrm{H}_{6} \mathrm{O}$ & 0.21 \\
\hline Benzothiazole & $\mathrm{S}$ & 613 & 530 & $\mathrm{C}_{7} \mathrm{H}_{5} \mathrm{NS}$ & -0.41 \\
\hline Vanillin & Food additive & 755.8 & 846 & $\mathrm{C}_{8} \mathrm{H}_{8} \mathrm{O}_{3}$ & -0.45 \\
\hline Diphenyl ether & Industrial chemical & 758.8 & 532 & $\mathrm{C}_{12} \mathrm{H}_{10} \mathrm{O}$ & 0.02 \\
\hline Dimethyl phthalate & Plasticiser & 787.7 & 891 & $\mathrm{C}_{10} \mathrm{H}_{10} \mathrm{O}_{4}$ & -0.55 \\
\hline
\end{tabular}


TABLE 5 (continued)

Selected compounds found in water from the Moreletaspruit by GC-HRT

\begin{tabular}{|l|l|c|c|c|c|}
\hline Compound & Class & RT (s) & Similarity & Formula & $\begin{array}{c}\text { Mass accuracy } \\
\text { (ppm) }\end{array}$ \\
\hline Cyclodecane & Hydrocarbon & 809.3 & 776 & $\mathrm{C}_{10} \mathrm{H}_{2} \mathrm{O}$ & 0.75 \\
\hline Benzylbenzoate & Plant constituent & 1015.3 & 823 & $\mathrm{C}_{14} \mathrm{H}_{12} \mathrm{O}_{2}$ & -0.86 \\
\hline Anthracene & PAH & 1027.5 & 834 & $\mathrm{C}_{14} \mathrm{H}_{10}$ & 0.5 \\
\hline Caffeine & Stimulant & 1065.9 & 570 & $\mathrm{C}_{8} \mathrm{H}_{10} \mathrm{~N}_{4} \mathrm{O}_{2}$ & 2.65 \\
\hline Fluoranthene & PAH & 1196.3 & 677 & $\mathrm{C}_{16} \mathrm{H}_{10}$ & -0.29 \\
\hline Methyl stearate & FAME & 1222 & 795 & $\mathrm{C}_{19} \mathrm{H}_{38} \mathrm{O}_{2}$ & -2.58 \\
\hline Flusizilazole & Fungicide & 1271.9 & 768 & $\mathrm{C}_{16} \mathrm{H}_{15} \mathrm{~F}_{2} \mathrm{~N}_{3} \mathrm{Si}$ & -3.26 \\
\hline
\end{tabular}

${ }^{1}$ Fatty acid methyl ester

\begin{tabular}{|c|c|c|c|c|c|}
\hline \multicolumn{6}{|c|}{$\begin{array}{l}\text { TABLE } 6 \\
\text { Selected compounds found in water from the Rietvleispruit by GC-HRT }\end{array}$} \\
\hline Compound & Class & $\mathbf{R T}(\mathbf{s})$ & Similarity & Formula & $\begin{array}{l}\text { Mass accuracy } \\
\quad(p p m)\end{array}$ \\
\hline 3-Methyl-2-butanone & Solvent & 175.1 & 712 & $\mathrm{C}_{5} \mathrm{H}_{10} \mathrm{O}$ & 0.1 \\
\hline 3-Hexanone & Solvent & 222.5 & 977 & $\mathrm{C}_{6} \mathrm{H}_{12} \mathrm{O}$ & 0.74 \\
\hline p-Xylene & Industrial chemical & 289.3 & 845 & $\mathrm{C}_{8} \mathrm{H}_{10}$ & 0.77 \\
\hline 2,5-Hexanedione & Diketone & 583 & 904 & $\mathrm{C}_{6} \mathrm{H}_{10} \mathrm{O}_{2}$ & 1.16 \\
\hline Benzaldehyde & Flavourant & 584 & 926 & $\mathrm{C}_{7} \mathrm{H}_{6} \mathrm{O}$ & -0.62 \\
\hline Butyrolactone & Recreational intoxicant & 668.8 & 852 & $\mathrm{C}_{4} \mathrm{H}_{6} \mathrm{O}_{2}$ & 1.18 \\
\hline 2-Furanmethanol & Industrial chemical & 692.3 & 640 & $\mathrm{C}_{5} \mathrm{H}_{6} \mathrm{O}_{2}$ & 1.42 \\
\hline 2-Decen-1-ol & Flavour and fragrance & 725 & 798 & $\mathrm{C}_{10} \mathrm{H}_{20} \mathrm{O}$ & -2.02 \\
\hline Diethylphthalate & Plasticiser & 1119.6 & 916 & $\mathrm{C}_{12} \mathrm{H}_{14} \mathrm{O}_{4}$ & -1.14 \\
\hline $\begin{array}{l}\text { 2-Ethylhexyl } \\
\text { trans-4-methoxycinnamate }\end{array}$ & Sunscreen and lip balm & 1155.5 & 741 & $\mathrm{C}_{18} \mathrm{H}_{26} \mathrm{O}_{3}$ & -0.3 \\
\hline 5-Hydroxymethylfurfural & Food constituent & 1187.4 & 656 & $\mathrm{C}_{6} \mathrm{H}_{6} \mathrm{O}_{3}$ & 1.79 \\
\hline Fluoranthene & $\mathrm{PAH}$ & 1199.3 & 943 & $\mathrm{C}_{16} \mathrm{H}_{10}$ & -0.23 \\
\hline Pyrene & PAH & 1326.5 & 916 & $\mathrm{C}_{16} \mathrm{H}_{10}$ & -0.02 \\
\hline 4-Hydroxybenzaldehyde & Flavourant & 1403.3 & 746 & $\mathrm{C}_{7} \mathrm{H}_{6} \mathrm{O}_{2}$ & -0.07 \\
\hline
\end{tabular}

In this study, the passive sampler was employed as an inexpensive and simple pollutant screening tool. However, contaminant concentrations may be derived by using performance reference compounds to determine in situ passive sampler sampling rates followed by conversion into water concentration of the analytes by means of silicone rubber specific partition coefficients (Yates et al., 2007; O'Hara, 2009; Booij and Smedes, 2010; Monteyne et al., 2013). The silicone rubber tubing passive sampler as a quantitative device will form part of a follow-up study.

Commercial passive samplers can be expensive (ITRC, 2005). In contrast, the miniature passive sampler was cheap, with the cost of manufacturing per sampler less than ZAR35 (ZAR7 for the sampling loop + ZAR25 for the stainless steel mesh basket). The passive sampler requires a minimum of storage space and can withstand transport over rough terrain which is not always the case for glass sample bottles.

\section{CONCLUSIONS}

The PDMS passive sampling device was cheap and easy to use. Enhanced sensitivity was achieved by solvent-free sample introduction. Each sampling site exhibited a different chemical profile depending on the source of input. Contaminants detected in surface water included caffeine, personal care products, pharmaceuticals, pesticides and PAHs.

\section{ACKNOWLEDGMENTS}

Elna Louw and Riaan Marais, Rietvlei Dam Nature Reserve, Pretoria, South Africa, for kindly granting access to one of the sampling points; Jack Cochran, RESTEK, for generous donation of GC columns and consumables, and the National Research Foundation (NRF) for financial support.

\section{REFERENCES}

BABUSHOK VI, LINSTROM PJ and ZENKEVICH G (2011) Retention indices for frequently reported compounds of plant essential oils. $J$. Phys. Chem. Ref. Data 40 (4) 1-47.

BALTUSSEN E, CRAMERS CA and SANDRA PJF (2002) Sorptive sample preparation - a review. Anal. Bioanal. Chem. 373 3-22.

BOOIJ K and SMEDES F (2010) An improved method for estimating in situ sampling rates of nonpolar passive samplers. Environ. Sci. Technol. 44 6789-6794. 
FERREIRA AP (2005) Caffeine as an environmental indicator for assessing urban aquatic ecosystems. Cad. Saúde Pública 21 (6)1884-1892.

ITRC (INTERSTATE TECHNOLOGY and REGULATORY COUNCIL) (2005) Technology overview of passive sampler technologies. DSP-4. Interstate Technology and Regulatory Council Authoring Team, Washington DC. URL: www.itrcweb.org (Accessed 11 February 2014).

MONTEYNE E, ROOSE P and JANSEN CR (2013) Application of a silicone rubber passive sampling technique for monitoring $\mathrm{PAH}$ and PCBs at 3 Belgian coastal harbours. Chemosphere $91390-398$.

NAUDÉ Y and ROHWER ER (2012) Two multidimensional chromatographic methods for enantiomeric analysis of $o, p$ '-DDT and $o, p$ '-DDD in contaminated soil and air in a malaria area of South Africa. Anal. Chim. Acta 730 120-126.
NAUDÉ Y, VAN ROOYEN MW and ROHWER ER (2011) Evidence for a geochemical origin of the mysterious circles in the Pro-Namib desert. J. Arid Environ. 75 446-456.

O'HARA S (2009) Silicone rubber passive samplers for water quality monitoring of persistent organic pollutants in the marine environment (Thesis). Paper 47. URL: http://arrow.dit.ie/scienmas/47 (Accessed 10 February 2014).

VERENTCHIKOV AN, YAVOR MI, HASIN YI and GAVRIK MA (2005) Multireflection planar time of flight mass analyser. I: An analyser for a parallel tandem spectrometer. Tech. Phys. 50 73-81.

VERENTCHIKOV AN, YAVOR MI, HASIN YI and GAVRIK MA (2005) Multireflection planar time of flight mass analyser. II: The high-resolution mode. Tech. Phys. 50 82-86.

YATES K, DAVIES I, WEBSTER L, POLLARD P, LAWTON L and MOFFATT C (2007) Passive sampling: partition coefficients for a silicone rubber reference phase. J. Environ. Monit. 9 1116-1121. 